\title{
Bisphosphonate-Associated Atypical Subtrochanteric Femur Fracture
}

\author{
Ely A. Wolin, Kevin P. Banks, and Penny J. Vroman \\ Department of Radiology, San Antonio Military Medical Center, Fort Sam Houston, Texas
}

\begin{abstract}
Bisphosphonates help prevent progressive bone mineralization loss and subsequent osteoporotic fractures. However, long-term bisphosphonate therapy paradoxically increases the risk of a unique injury called an atypical subtrochanteric femur fracture. Despite this, the benefits of bisphosphonates outweigh the risks, because far more pathologic fractures are prevented than induced. The early identification of atypical subtrochanteric femur fractures is important as there is high associated morbidity and mortality. We describe a case of a 76-y-old woman with a completed bisphosphonate-associated atypical subtrochanteric femur fracture.
\end{abstract}

Key Words: bisphosphonate; osteoporosis; atypical subtrochanteric femoral fracture

J Nucl Med Technol 2015; 43:72-73

DOI: 10.2967/jnmt.114.139923

$\mathbf{L}$

ong-term bisphosphonate therapy is associated with an increased risk of atypical femoral fractures. Early identification and intervention is essential to avoid the associated morbidity and mortality of a completed fracture.

\section{CASE REPORT}

A 76-y-old woman presented with severe, atraumatic, right hip and thigh pain. She reported a history of osteopenia treated with bisphosphonates (nearly $4 \mathrm{y}$ of alendronate, followed by $4 \mathrm{y}$ of ibandronate). Femur radiographs at presentation were read as normal but on retrospect demonstrated focal cortical thickening along the lateral diaphysis (Fig. 1). A bone scan $5 \mathrm{~d}$ later showed focal radiotracer uptake at the same site, interpreted as concerning for an atypical subtrochanteric femur fracture (Fig. 2). Six days later, the patient re-presented with increasing pain after a fall from standing. Repeated radiographs showed a displaced transverse fracture through the proximal right femoral diaphysis (Fig. 3).

Received Mar. 7, 2014; revision accepted Apr. 28, 2014.

For correspondence or reprints contact: Ely Wolin, 3551 Roger Brooke Dr., Fort Sam Houston, TX 78234.

E-mail: ely.a.wolin.mil@mail.mil

Published online Aug. 14, 2014.

COPYRIGHT (c) 2015 by the Society of Nuclear Medicine and Molecular Imaging, Inc.

\section{DISCUSSION}

Osteoporosis places individuals at high risk for insufficiency and fragility fractures. Treatment includes lifestyle modifications and pharmacologic intervention, frequently with bisphosphonates (1).

Bisphosphonates work by inducing osteoclast apoptosis $(2,3)$. A known risk of long-term bisphosphonate therapy is a unique fracture known as an atypical subtrochanteric femur fracture. These may result from impaired remodeling allowing microfractures to progress (4). A 2008 study of the entire population of Sweden reported an age-adjusted relative

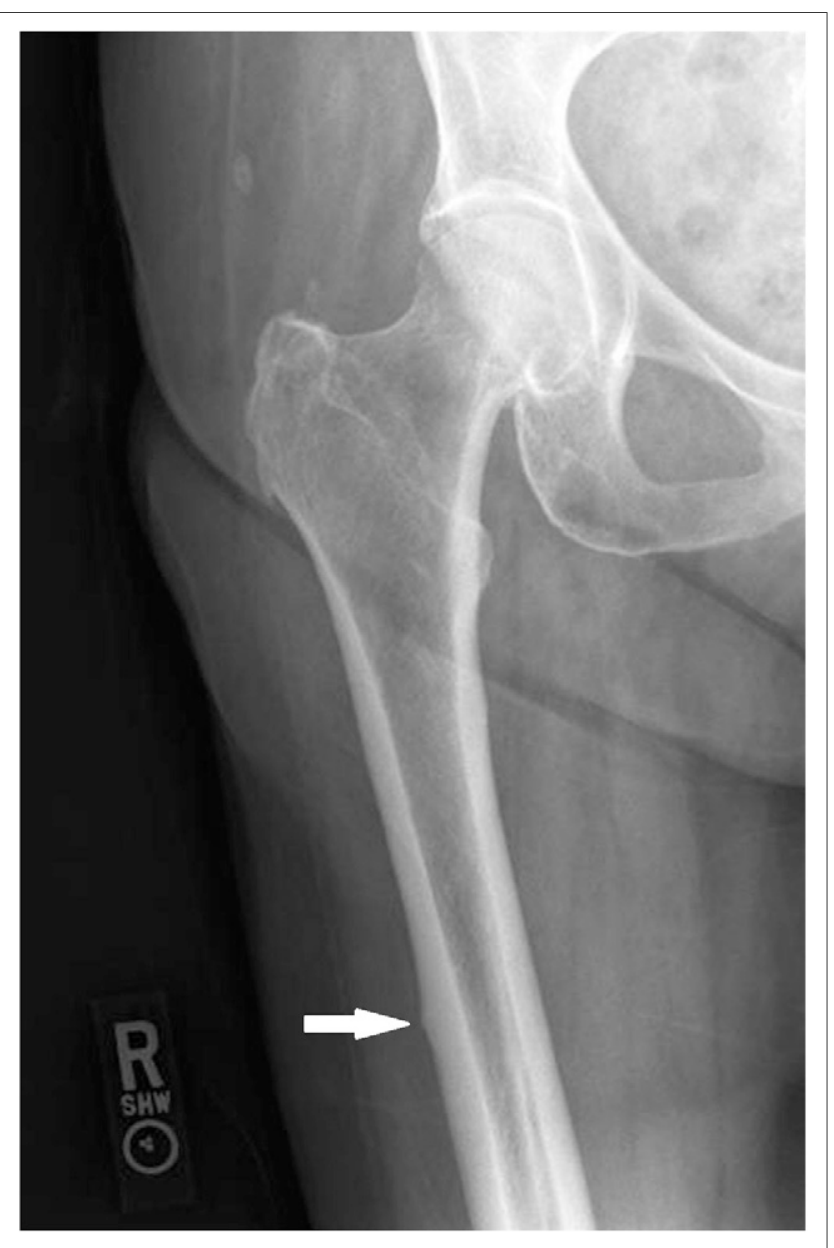

FIGURE 1. Small focal area of cortical thickening (arrow) along lateral cortex of proximal right femoral diaphysis. 


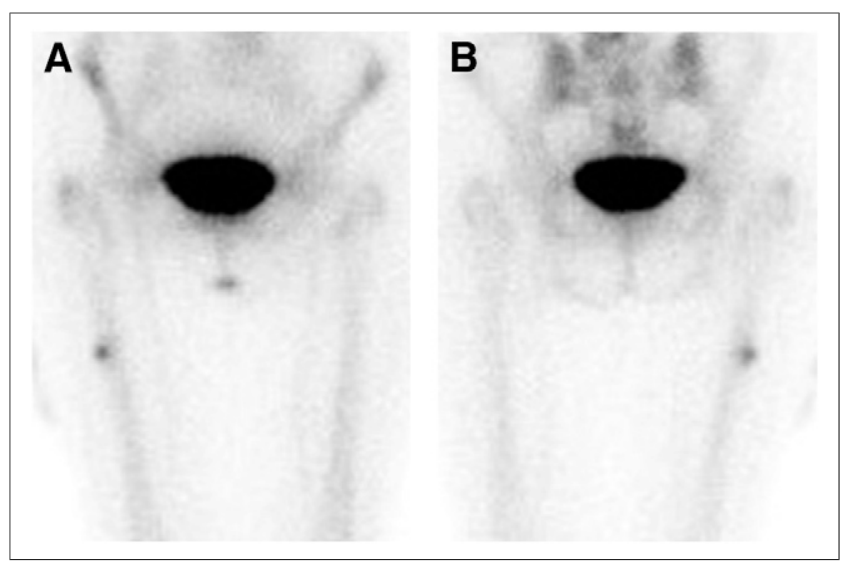

FIGURE 2. Anterior (A) and posterior (B) images from ${ }^{99 m T c-}$ labeled methyl diphosphonate bone scan demonstrating focal intense increased radiotracer uptake along lateral cortex of proximal right femoral diaphysis.

risk of atypical femur fracture with any bisphosphonate use of 47.3, corresponding to an additional 5 cases per 10,000 patient-years (5). Multiple additional epidemiologic studies showed a patient on bisphosphonates had a relative risk of atypical femur fracture ranging from 2.11 to 66.9 , although the absolute risk remains low (4). These fractures have a mortality rate of $14 \%$ at $12 \mathrm{mo}$ and $25 \%$ at $24 \mathrm{mo}(6)$.

The American Society for Bone and Mineral Research task force recommended a fracture must be located distal to the lesser trochanter to just proximal to the supracondylar flare and have 4 of these 5 major features to be classified as atypical: (1) no or minimal associated trauma, as in a fall from standing; (2) the fracture line originating at the lateral cortex, substantially transverse in its orientation; (3) complete fractures involving both cortices (may be associated with a medial spike) or incomplete fractures involving only the lateral cortex; (4) noncomminuted or minimally comminuted; and (5) localized periosteal or endosteal thickening of the lateral cortex is present (4). On bone scanning, there will be focal increased radiotracer activity corresponding to the area of cortical thickening (7).

Early diagnosis of incomplete fractures helps prevent completion by allowing for prophylactic operative management (4).

\section{CONCLUSION}

Although bisphosphonate therapy prevents far more osteoporotic fractures than it induces atypical fractures, atypical subtrochanteric fractures have high morbidity and mortality. Thus, early recognition is necessary to allow for appropriate protective operative intervention.

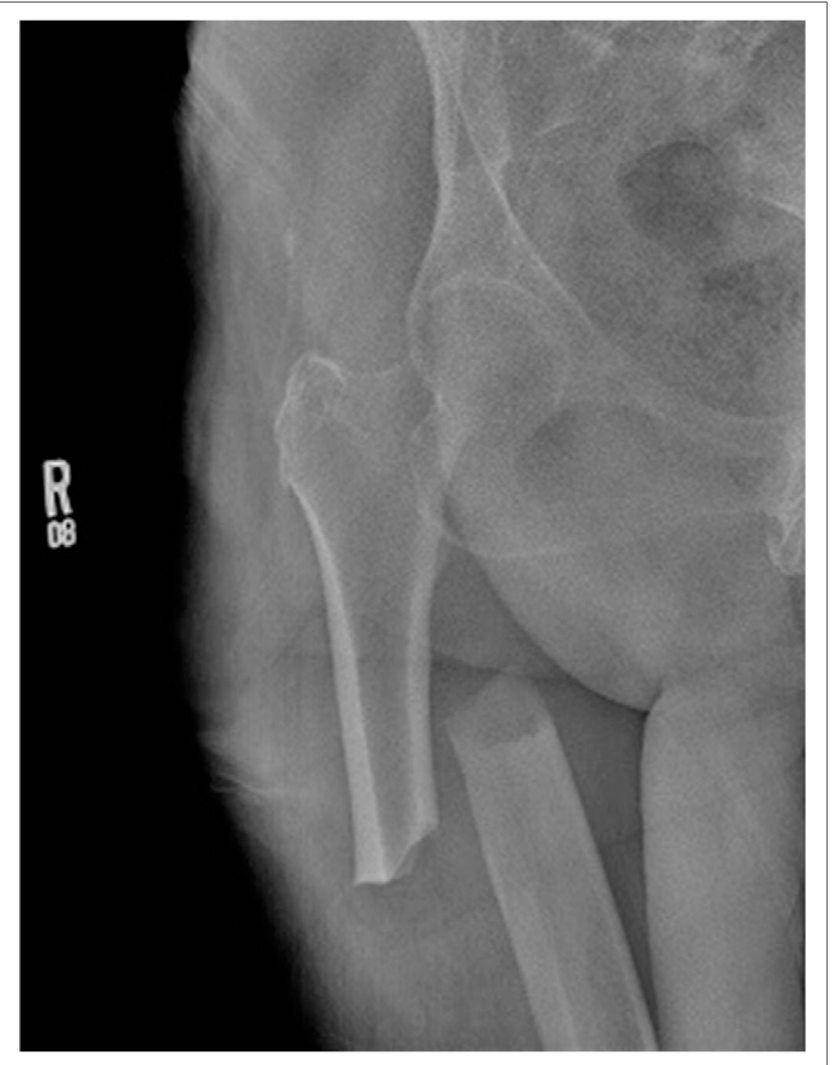

FIGURE 3. Markedly displaced transverse fracture of proximal right femoral diaphysis with bayoneting.

\section{DISCLOSURE}

No potential conflict of interest relevant to this article was reported.

\section{REFERENCES}

1. Rosen HN, Drezner MK. Overview of the management of osteoporosis in postmenopausal women. UpToDate. http://www.uptodate.com/contents/overview-ofthe-management-of-osteoporosis-in-postmenopausal-women. Updated January 24, 2014. Accessed July 11, 2014.

2. Rosen HN. The use of bisphosphonates in postmenopausal women with osteoporosis. UpToDate. http://www.uptodate.com/contents/the-use-of-bisphosphonates-inpostmenopausal-women-with-osteoporosis. Updated September 19, 2013. Accessed July 11, 2014.

3. Chan SS, Rosenberg ZS, Chan K, Capeci C. Subtrochanteric femoral fractures in patients receiving long-term alendronate therapy: imaging features. AJR. 2010; 194:1581-1586

4. Shane E, Burr D, Abrahamsen B, et al. Atypical subtrochanteric and diaphyseal femoral fractures: second report of a task force of the American Society for Bone and Mineral Research. J Bone Miner Res. 2014;29:1-23.

5. Schilcher J, Michaelsson K, Aspenberg P. Bisphosphonate use and atypical fractures of the femoral shaft. N Engl J Med. 2011;364:1728-1737.

6. Ekström W, Nemeth G, Smnegard E, Dalen N, Tidermark J. Quality of life after a subtrochanteric fracture: a prospective cohort study on 87 elderly patients. Injury. 2009;40:371-376.

7. Probst S, Rakheja R, Stern J. Aytpical bisphosphonate-associated subtrochanteric and femoral shaft stress fractures. Clin Nucl Med. 2013;38:397-399. 